Tortosa Cuesta, Rubén; Sánchez López, Miguel; Ávila González, Mireia; Azevedo Monteiro, Bruno Miguel 4xiDraw Máquina de visualización de datos: la imagen creada desde la información como práctica artística IV Congreso INTERNACIONAL DE INVESTIGACIÓN EN ARTES VISUALES ANIAV 2019 IMAGEN [N] VISIBLE]

http://dx.doi.org/10.4995/ANIAV.2019.9588

Tortosa Cuesta, Rubén.

Profesor Titular de Universidad, Universitat Politècnica de València, Departamento de Dibujo.

Sánchez López, Miguel.

Profesor Titular de Universidad, Universitat Politècnica de València, Departamento de Informática de Sistemas y Computadores.

Ávila González, Mireia.

Alumna doctorado, Universitat Politècnica de València.

Azevedo Monteiro, Bruno Miguel.

Universidade do Minho. Portugal.

\title{
4xiDraw Máquina de visualización de datos: la imagen creada desde la información como práctica artística
}

\section{4xiDraw Data visualization machine: the image created from information as an artistic practice}

TIPO DE TRABAJO: Póster.

PALABRAS CLAVE

Datos, visualización, máquina, digital, arte, dibujo.

KEY WORDS

Data, visualization, machine, digital, art, drawing.

RESUMEN

Planteamos la construcción de la imagen a partir de los Datos como materia prima, en la que intervienen la mezcla e hibridación de lenguajes. El paso de lo analógico a lo digital y vuelta a lo analógico, es decir, de un código, de un signo a otro, nos abre la posibilidad de múltiples lecturas de lo traducido, para dar sentido a la imagen desde dispositivos de memoria externa. Éste es un hecho caracterizado por las transposiciones, formatos, materiales, escalas, traslaciones y transcodificaciones. En definitiva, nomadismo entre los procesos.

\footnotetext{
La imagen generada mediante dispositivos digitales, ahora es tiempo simultáneo. No somos espectadores sino parte del proceso, en el que la realidad se transforma en representación. En este sentido, habríamos de añadir la hibridación temporal a las teorías contemporáneas de la hibridación.
}

Para la realización de la obra artística The Bird: the journal of the image, resultado de esta investigación, tomamos los Datos como punto de partida en lo que será el primer acto de creación. En un segundo acto, estos datos junto a la máquina (hardware y software) como elementos físico y virtual, harán posible visualizarlos en dibujo (imagen). La máquina 4xiDraw ha sido diseñada ex profeso para hacer posible la traducción de la información. En este caso la obtenemos de la señal que transmite un geo-localizador colocado a un cernícalo que pertenece a un programa de protección de especies en extinción, cuyos desplazamientos se traducen en un trazado de líneas sobre una hoja de papel de $70 \times 100 \mathrm{~cm}$, a modo de diario. La obra se denomina The Bird: the journal of the image.

Las piezas, para ensamblar 4xiDraw, han sido diseñadas en software CAD e impresas mediante impresora 3D en PLA. Para su construcción se han utilizado: dos motores paso a paso Nema 17, 2 varillas de acero de 1000x8 mm, 2 varillas roscadas de acero de 
Tortosa Cuesta, Rubén; Sánchez López, Miguel; Ávila González, Mireia; Azevedo Monteiro, Bruno Miguel 4xiDraw Máquina de visualización de datos: la imagen creada desde la información como práctica artística IV Congreso INTERNACIONAL DE INVESTIGACIÓN EN ARTES VISUALES ANIAV 2019 IMAGEN [N] VISIBLE] http://dx.doi.org/10.4995/ANIAV.2019.9588

1000×10 mm, servo, correa dentada, 2 poleas GT2, RaspberryPi, electrónica, fuentes de alimentación, pantalla de 3,5", tinta pigmentada y papel.

\section{ABSTRACT}

We propose to construct the image taking Data as raw material, in which the blend and hybridization of languages take place. The transition from the analogic to digital and vice-versa, that is to say, from one code or sign to another, opens up a possibility of multiple meanings so the image translated makes sense from external memory supports. This is a fact characterized by the transpositions, formats, materials, scales, metaphors and transcodings. Ultimately, nomadism between processes.

The imaged generated through digital devices, is now simultaneous time. Now, we are not spectators but part of the process, where reality becomes representation. Accordingly, we would have to add the temporal hybridization to the contemporary hybridization theories.

To carry out the artwork The Bird: The Journal of the Image, which is the research results, we take Data as a starting point to give rise to the first act of creation. In a second act, this Data along with the machine (hardware and software) as virtual and material elements, will make possible to visualize them as a drawing (image). The machine, 4xiDraw has been designed ad hoc to bring the Data translation forth. In this case, we obtain the information from a signal transmitted by a geo-locator ring banded to a kestrel's leg, part of a protection of endangered species program, which journey is translated into a layout on a $70 \times 100 \mathrm{~cm}$ sheet of paper as a sort of diary.

The pieces to assemble the 4xiDraw machine have been designed in CAD software and printed with a 3D printer with PLA material. To build this machine we have used two step motors Nema 17, two $1000 \times 8 \mathrm{~mm}$ steel rods, two $1000 \times 10 \mathrm{~mm}$ threaded rods, a servo, a toothed belt, two pulleys GT2, a Raspberry Pi, electronics, power supply sources, 3,5" screen, pigment ink and paper.

\section{INTRODUCCIÓN}

Consideramos que la información Datos, es la "materia prima". De este modo, ella y los flujos informacionales constituyen la expresión/recurso/materia dominante de la actual sociedad. Estos Datos los consideramos el primer acto de creación. Por otro lado, partiendo del concepto $\mathrm{Maker}^{1}$, hemos desarrollado una máquina de dibujo denominado 4xiDraw (Figura 1), para convertir y visualizar la información recogida del trayecto diario de un cernícalo (Falco tinnunculus) protegido. El ordenador traducirá los datos en movimiento, para dibujar sobre una hoja de papel de $70 \times 100 \mathrm{~cm}$ el recorrido del ave (Figura 2), en lo que será el segundo acto de creación que conformará el acontecimiento.

La aparición de un aparato de ver - o quizás deberíamos decir: de una máquina capaz de capturar y reproducir imágenes mecánicamenteconstituye un auténtico acontecimiento, una modificación tan decisiva que con ella el ver -y toda la culturalidad que le concierne- se desplaza a un nuevo escenario, radicalmente redefinido. Ver, ciertamente ya no será lo mismo. Variará, además, el conjunto global de las reglas que ordenan los modos del representar, del producir imágenes (...) Para todo ello, la aparición del ojo técnico resulta fundamental. Él, la máquina de ver y producir técnicamente las imágenes que de ese ver resultan, traerá modificaciones que acabarán por 'prefigurar un orden de la visión radicalmente diferenciado. Brea (2010)

La visualización de la información, opera transversalmente en una conexión entre el mundo tangible y el mundo intangible. La visualización de la información se caracteriza como un proceso de convertir datos en imágenes.

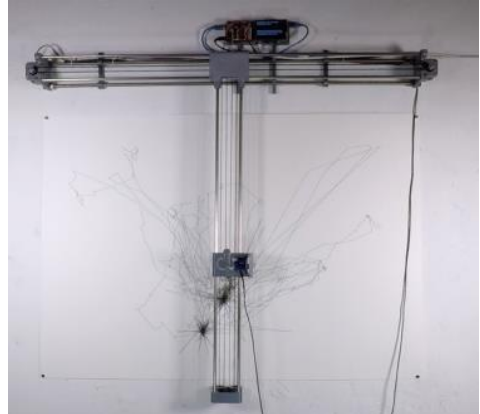

Figura 1. Máquina de dibujo 4xiDraw

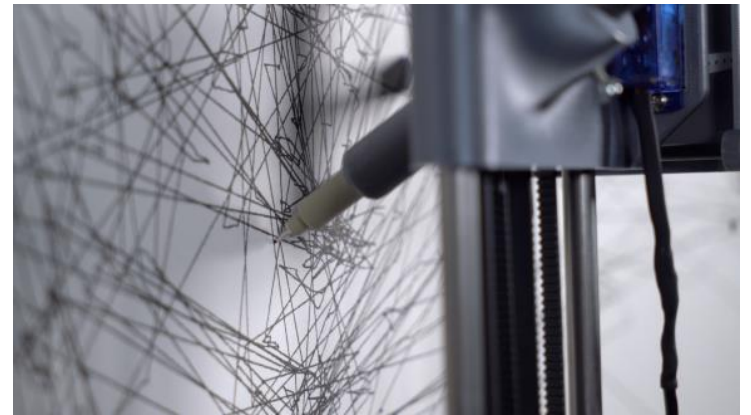

Figura 2. Dibujo papel. detalle

${ }^{1}$ https://es.wikipedia.org/wiki/Cultura maker (última consulta 25 de marzo de 2019) 
Tortosa Cuesta, Rubén; Sánchez López, Miguel; Ávila González, Mireia; Azevedo Monteiro, Bruno Miguel 4xiDraw Máquina de visualización de datos: la imagen creada desde la información como práctica artística IV Congreso INTERNACIONAL DE INVESTIGACIÓN EN ARTES VISUALES ANIAV 2019 IMAGEN [N] VISIBLE] http://dx.doi.org/10.4995/ANIAV.2019.9588

\section{METODOLOGÍA}

A través de la asociación Xaloc y de su director Carles Gago, se nos ha proporcionado la información de varios años que abarca diferentes ejemplares de cernícalo, dentro de la misma reserva, al que se le está realizando seguimiento para su estudio de hábitos, hábitat y control de protección. Los datos consisten en ubicaciones de latitud y longitud con una firma de tiempo y el identificador del ave, que nos permite conocer la ubicación de un ejemplar concreto en un momento concreto. Una vez se han ordenado estos datos, se ha grabado en la memoria del ordenador. Por otra parte, para la realización de la máquina de dibujar denominada 4xiDraw, se han diseñado, mediante software Onshape, todas las piezas necesarias para ser impresas mediante impresora 3D modelo Prusa MK3, con PLA gris. Estas piezas se han testado y rediseñado en base a las pruebas realizadas. Finalmente se ha montado el dispositivo y se ha conectado todos los componentes.

\section{DESARROLLO}

Para confeccionar los recorridos que muestra "The Bird", se han seleccionado ejemplares y días concretos que han dado lugar a cada uno de los recorridos individuales que se van trazando. Cada trazo, que consta de múltiples segmentos rectos que unen las diferentes ubicaciones secuenciales registradas de ese ejemplar en ese día concreto. Al efecto de representar el comportamiento de un modo más preciso, pero también sin perder dinamismo, las pausas que puede realizar el ave en su recorrido diario también se reflejan, a una escala de tiempo acelerada, en el sistema de trazado. (Figura 2)

La información se ha elaborado guardándola en una base de datos open-source SQLite3 que luego se ha tratado mediante diferentes consultas SQL y pequeños shell scripts para obtener una representación del recorrido basada en el formato de los archivos G-code empleados por la tarjeta Arduino que emplea el programa open-source GRBL. El trabajo de la Raspberry Pi, que ejecuta una distribución "Jesse" de Linux, lo realizan varios programas ad-hoc escritos en Java y Python. (Figura 3)

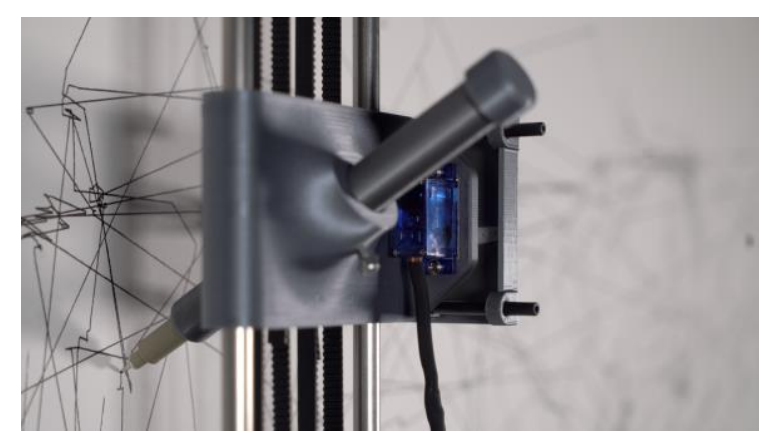

Figura 2 Eje $Y$ donde se aloja el sistema de dibujo

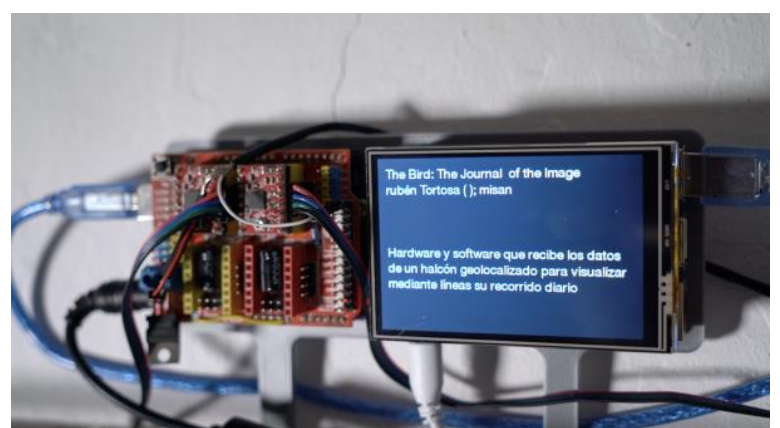

Figura 3 RaspberryPi, Pantalla, Arduino y electrónica

La información,se ha dispuesto de manera que puede ser utilizada tanto online como offline, dependiendo del lugar de la instalación de la obra.

Mediante software Onshape, se han diseñado las piezas del dispositivo. Estas piezas se han impreso en PLA gris con impresora 3D Prusa MK3 y MK2 (figura 4, 5). Una vez impresas las piezas, se ha ensamblado y añadido el resto de componentes formado por:

- Raspberry Pi y fuente de alimentación

- $\quad$ Arduino UNO y fuente de alimentación

- Pantalla táctil 3,5 Pulgadas HD 1920×1080

- $\quad$ Memoria micro SD 16 GB

- Electrónica y drivers

- $\quad$ Cable USB 
Tortosa Cuesta, Rubén; Sánchez López, Miguel; Ávila González, Mireia; Azevedo Monteiro, Bruno Miguel 4xiDraw Máquina de visualización de datos: la imagen creada desde la información como práctica artística IV Congreso INTERNACIONAL DE INVESTIGACIÓN EN ARTES VISUALES ANIAV 2019 IMAGEN [N] VISIBLE] http://dx.doi.org/10.4995/ANIAV.2019.9588

- 2 motores Nema 17

- 4 varillas de acero calibrado de 1000 mm M8

- 2 varillas de acero roscado de $1000 \mathrm{~mm}$ M10

- 1 microservo SG 90

- 4 metros de correa dentada GT2

- 8 tuercas M10

- $\quad 2$ poleas dentadas y 7 poleas lisas

- 18 tornillos $\mathrm{m} 8$

- $\quad 4$ m de cable

- $\quad$ Rotulador tinta de archivo Sakura

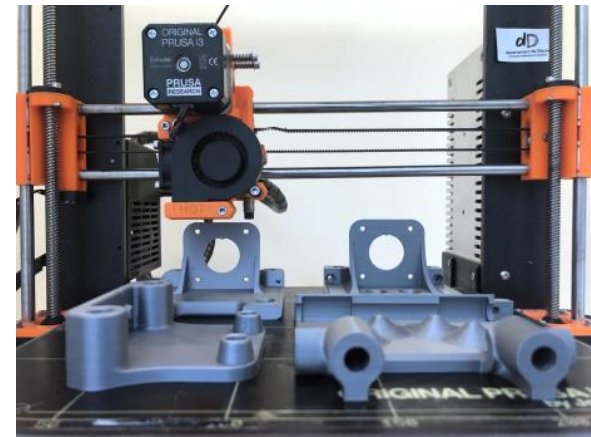

Figura 4 Impresión piezas ensamblaje. Impresora 3D

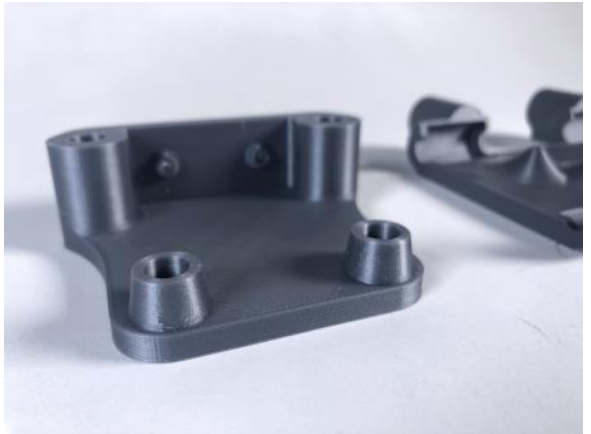

Figura 5 Detalle piezas impresas 3D PLA

\section{CONCLUSIONES}

La obra The Bird: the journal of the image, propone la creación de la obra artística mediante la visualización de datos. Para ello se ha recurrido a utilización de software libre y diseñado y construido una máquina de dibujo ad hoc denominada 4xiDraw. Se ha utilizado impresora 3D, en un sistema de trabajo Maker. Hemos recurrido a nuevo proceso de diseño y montaje del dispositivo sin tener que depender de la industria, lo que ha facilitado las pruebas y mejoras a un coste de producción muy bajo. Las piezas 3D se pueden descargar de manera gratuita y pueden ser utilizadas por otros artistas para la construcción de esta máquina. Todo ello constata un método de trabajo, en el que se comparte el conocimiento. En el que la obra se realiza a partir de visualizar datos, ya no necesitamos una imagen concreta. Una máquina, relativamente económica, construida por uno mismo visualiza estos datos en forma de dibujo.

\section{FUENTES REFERENCIALES}

Brea, J. L. (2010). Las tres eras de la imagen. Madrid, España: Akal. 